\title{
Chimiokines, VIH et médias
}

RANTES, MIP- $1 \alpha$, MIP-1 $\beta$ : les molécules qui se cachent derrière ces mystérieux acronymes seraient-elles des antiviraux naturels, capables de bloquer la réplication du rétrovirus du SIDA (VIH) ? C'est ce qu'affirment les équipes de Paolo Lusso et Robert Gallo dans un article publié par Science et intensément médiatisé [1]. On sait que le VIH se propage dans la sous-population lymphocytaire qui exprime le marqueur $\mathrm{CD} 4$, et non dans celle qui exprime CD8. Dès 1986, Jay Levy et ses collaborateurs avaient observé que la propagation de VIH était moins efficace quand les lymphocytes $\mathrm{CD}^{+}{ }^{+}$étaient mis en coculture avec des lymphocytes CD8 ${ }^{+}$ provenant de sujets infectés. Cet effet semblait dû à la sécrétion de facteurs "suppresseurs» par les lymphocytes $\mathrm{CD}^{+}$, puisqu'il était reproduit par leur milieu de culture filtré [2].

Depuis cette observation initiale, les facteurs suppresseurs furent activement recherchés, mais ne purent être identifiés. Cocchi et al. y seraient parvenus, par une approche assez classique consistant à fractionner au maximum le surnageant d'une lignée lymphocytaire $\mathrm{CD} 8^{+}$, et à rechercher par microséquençage quelles protéines étaient présentes dans les fractions contenant l'activité suppressive. Ils ont ainsi détecté trois molécules apparentées, RANTES, MIP-1 $\alpha$ et $\beta$, des cytokines produites au cours de la réponse inflammatoire et jouant, entre autres, un rôle chimiotactique, d'où leur nom de chimiokines [3]. Bien sûr, leur présence dans les fractions «suppressives" n'était pas suffisante, et différentes expériences amènent les auteurs à affirmer que ces chimiokines sont bien les facteurs suppresseurs. Des chimiokines re- combinantes inhibent de façon dépendante de la dose la propagation de VIH dans la lignée PM1, quoique cet effet soit plus ou moins prononcé selon les isolats, certains étant totalement résistants. Par ailleurs, l'activité suppressive sécrétée par les lymphocytes $\mathrm{CD}^{+}$peut être neutralisée par une combinaison de trois anticorps dirigés contre RANTES, MIP- $1 \alpha$ et MIP-1 $\beta$. Testés isolément, ces anticorps n'ont pas d'effet, ce qui amène les auteurs à suggérer une synergie d'action des trois chimiokines.

On peut rapprocher ce travail d'une observation plus préliminaire, récemment publiée par Nature sous forme de correspondance scientifique [4]. Une autre cytokine, l'interleukine 16 (IL-16), réduirait de façon spectaculaire la propagation de VIH dans des cultures de lymphocytes. Curieusement, seule l'IL-16 de singe vert africain est active, et non l'IL-16 humaine pourtant très proche.

Ces différentes observations ont suscité des réserves. Cité dans un éditorial publié par Science une semaine avant le travail de Cocchi et al., Jay Levy affirme avoir, au cours de sa quête du facteur suppresseur, testé et éliminé RANTES, MIP- $1 \alpha$ et $\beta$, ainsi que l'IL-16 [5]. Les raisons de cette discordance avec les résultats de l'équipe de Gallo ne sont pas évidentes, mais les virologues savent que des effets minimes sur la cellule, et en particulier sur sa cinétique de réplication, peuvent entraîner des différences spectaculaires de propagation virale. On peut donc se demander si la lignée PM1 n'est pas particulièrement sensible aux effets des chimiokines, et regretter que d'autres cellules n'aient été testées. Par ailleurs, on ne sait pas dans quel- le mesure les facteurs suppresseurs, chimiokines ou autres, identifiés par des tests cellulaires, peuvent contribuer au contrôle de l'infection in vivo. Même en leur supposant une certaine efficacité, il est malheureusement clair qu'ils ne peuvent enrayer l'évolution de l'infection par VIH.

Il semble donc prématuré d'affirmer que les chimiokines sont douées de propriétés antivirales, et regrettable que ces molécules soient présentées au public comme des médicaments potentiels. Fort heureusement, ledit public a appris depuis longtemps à se méfier des grands espoirs de traitement du SIDA claironnés à tout propos par les médias. Ceux-ci feraient un travail plus utile en rappelant qu'il existe des antiviraux dont l'efficacité sur VIH est directe et avérée, et que l'association de ces molécules représente un réel espoir thérapeutique.

M.A.

1. Cocchi F, DeVico AL, Garzino-Demo A, Arya SK, Gallo RC, Lusso P. Identification of RANTES, MIP- $1 \alpha$ and MIP- $1 \beta$ as the major HIV-suppressive factors produced by CD8 ${ }^{+}$T cells. Science 1995 ; 270: $1811-5$

2. Walker CM, Moody DJ, Stites DP, Levy JA. CD8 ${ }^{+}$ lymphocytes can control HIV infection in vitro by suppressing virus replication. Science 1986; 234 : 1563-6.

3. Minty A. Une nouvelle famille de cytokines inflammatoires. médecine/sciences 1991; 7: 578-88.

4. Baier M, Werner A, Bannert N, Metzner K, Kurth R. HIV suppression by interleukin-16. Nature $1995 ; 378: 563$.

5. Balter M. Elusive HIV-suppressor factors found. Science 1995 ; 270 : 1560-1. 